\title{
The Exome Clinic and the role of medical genetics expertise in the interpretation of exome sequencing results
}

\author{
Dustin Baldridge, MD, PhD', Jennifer Heeley, MD ${ }^{1,4}$, Marisa Vineyard, MS, CGC1, \\ Linda Manwaring, MS, CGC ${ }^{1}$, Tomi L. Toler, MS, CGC ${ }^{1}$, Emily Fassi, MS, CGC ${ }^{1}$, Elise Fiala, MS, CGC ${ }^{1}$, \\ Sarah Brown, PhD², Charles W. Goss, PhD³ ${ }^{3}$ Marcia Willing, MD, PhD ${ }^{1}$, Dorothy K. Grange, MD', \\ Beth A. Kozel, MD, PhD ${ }^{1,5}$ and Marwan Shinawi, MD ${ }^{1}$
}

Purpose: Evaluation of the clinician's role in the optimal interpretation of clinical exome sequencing (ES) results.

Methods: Retrospective chart review of the first 155 patients who underwent clinical ES in our Exome Clinic and direct interaction with the ordering geneticist to evaluate the process of interpretation of results.

Results: The most common primary indication was neurodevelopmental problems $(\sim 66 \%)$, followed by multiple congenital anomalies $(\sim 10 \%)$. Based on sequencing data, the overall diagnostic yield was $36 \%$. After assessment by the medical geneticist, incorporation of detailed phenotypic and molecular data, and utilization of additional diagnostic modalities, the final diagnostic yield increased to $43 \%$. Seven patients in our cohort were included in initial case series that described novel genetic syndromes, and $23 \%$ of patients were involved in subsequent research studies directly related to their results or involved in efforts to move beyond clinical ES for diagnosis. Clinical management was directly altered due to the ES findings in $12 \%$ of definitively diagnosed cases.

Conclusions: Our results emphasize the usefulness of ES, demonstrate the significant role of the medical geneticist in the diagnostic process of patients undergoing ES, and illustrate the benefits of postanalytical diagnostic work-up in solving the "diagnostic odyssey."

Genet Med advance online publication 2 March 2017

Key Words: diagnostic yield; Exome Clinic; exome sequencing; genetic counseling; medical geneticist

\section{INTRODUCTION}

Clinical exome sequencing (ES) has revolutionized the diagnostic work-up for patients with genetic disease and has changed the diagnostic process in medical genetics practice. ${ }^{1}$ The increasing utilization of ES has rapidly identified new genetic syndromes and has contributed to solving many diagnostic odysseys. ${ }^{2}$ Reports of the yield of exome sequencing through diagnostic laboratories have ranged from 25 to $30 \% .^{3-5}$ Trio sequencing and focusing on specific disease subgroups can raise the diagnostic rate. ${ }^{5,6}$ Many (23-30\%) of these diagnosed patients were found to have mutations in genes that had been reported in association with the respective phenotype within the prior 2 to 3 years. ${ }^{3,5}$

Exome sequencing has provided insights into the genetic and phenotypic heterogeneity (e.g., atypical and milder presentations) of Mendelian disorders and highlighted the importance of de novo mutations and "blended phenotypes" (co-existing diagnoses that combine the clinical features of each) in rare genetic disorders. ${ }^{3-5}$ The application of this unbiased wholegenome technology has led to shifting of the diagnostic skills of the medical geneticist from focusing on detailed phenotypic characterization to identifying the genetic etiology to "nextgeneration phenotyping," which involves interpretation and validation of molecular test results in clinical practice by analyzing observed clinical features.

To date, only a few attempts have been made to study the role played by the medical geneticist in the interpretation of results as part of the diagnostic process of ES, the concordance rate between the laboratory exome results and the geneticist's interpretation, and the ability of ES to alter a patient's or family's medical management. Duke recently reported that medical geneticists and laboratories were $90 \%$ concordant in their interpretation of the exome results and that discordance occurred when the medical geneticist reconsidered additional clinical information and/or additional laboratory tests and genotyping of family members. ${ }^{8}$ Another study showed that establishing a diagnosis through ES can lead to discontinuation of additional planned studies, screening patients for additional manifestations, altering management, identification of disease in other at-risk family members, and reproductive planning. ${ }^{9}$ The

\footnotetext{
${ }^{1}$ Division of Genetics and Genomic Medicine, Department of Pediatrics, Washington University School of Medicine, St. Louis, Missouri, USA; ${ }^{2}$ Department of Pathology and Immunology, Washington University School of Medicine, St. Louis, Missouri, USA; ${ }^{3}$ Division of Biostatistics, Washington University School of Medicine, St. Louis, Missouri, USA; ${ }^{4}$ Current affiliation: Mercy Clinic_-Kids Genetics, Mercy Children's Hospital St. Louis, St. Louis, Missouri, USA; ${ }^{5}$ Current affiliation: National Heart, Lung, and Blood Institute, National Institutes of Health, Bethesda, Maryland, USA. Correspondence: Marwan Shinawi (Shinawi_M@kids.wustl.edu)
} 
potential cost-effectiveness of ES has also been evaluated by calculating the cost of previous diagnostic workups, concluding that in some cases it may be most cost-effective to perform ES as a first test. ${ }^{10}$

In this study, we present our experience with the "Exome Clinic" with special emphasis on the diagnostic course after ES has been completed by the laboratory. We evaluate the role of the medical geneticist in the interpretation of results, auxiliary studies performed to determine pathogenicity of genetic variants, follow-up clinical tests, and postexome enrollment in research studies. We discuss the diagnostic yield of ES in our cohort as a function of different phenotypic features. The utility of exome reanalysis 1-2 years after the original report is also presented. Finally, we have recorded details of the social and financial implications of our exome results, such as determinations of misattributed paternity and the patient's out-of-pocket cost.

\section{MATERIALS AND METHODS}

\section{Chart review and clinical evaluation}

The Washington University School of Medicine Institutional Review Board approved this study. Clinical data were obtained by retrospective chart review and interview with the ordering medical geneticists and genetic counselors (Supplementary Material 1 online).

\section{ES Laboratory Results}

Exomes for 155 probands were ordered between March 2012 and January 2015. Exomes were performed in three laboratories: 127 were analyzed through GeneDx (Gaithersburg, MD), 20 were analyzed through Ambry Genetics (Aliso Viejo, CA) and 8 were analyzed through Baylor Genetics (Houston, TX). Laboratories reported genetic variants as pathogenic, likely pathogenic, or variants of uncertain significance (VUS) but did not report benign or likely benign variants. We refer to this classification as variant-level assertion. GeneDx also classified the variants in relation to the patient's phenotype as either definitively or possibly related and reported potential candidate genes for new genetic syndromes, which had not previously been associated with a human phenotype. Ambry Genetics classified variants as either likely positive, which we interpreted as possible, or positive, which we considered as definitively associated with the phenotype. Baylor Genetics classified the variants under "disease genes related to clinical phenotype" as either "deleterious" or "VUS." We considered "deleterious" and "VUS" as definitive and possible, respectively. All three laboratories also reported incidental variants. Definitions of these terms were adapted from Retterer et al. ${ }^{6}$ We refer to these definitive, possible, candidate, and incidental classifications as case-level assertion, which is a synthesis of all the molecular data in a single subject specifying whether the test results provide a molecular diagnosis according to the testing laboratory.

\section{Clinical assessment of ES findings}

Results of ES were discussed individually with the ordering medical geneticist and exome findings were confirmed or reclassified as needed as definitively, likely, possibly, or unlikely causative of the patient's symptoms based on the molecular data (variant and case-level classifications) and the geneticist's clinical assessment (Supplementary Material 1 online). We refer to this classification as clinical-level assertion. This clinical impression was then categorized as concordant or discordant with the laboratory's case-level assertion to allow us to analyze how the geneticist's interpretation influenced the final diagnosis (Supplementary Material 1 online). The statistical tools used for data analysis are presented in Supplementary Material 1 online.

\section{Characteristics of the cohort}

\section{RESULTS}

Detailed descriptions of the clinical characteristics and molecular findings of the patients are documented in Supplementary Detailed Data Table online. Demographic and phenotypic characteristics of our cohort are recorded in Table 1 and Supplementary Material 1 online. Sequencing costs for Medicaid patients were not covered by their insurance plans and

\section{Table 1 Demographic cohort details}

\begin{tabular}{|c|c|}
\hline \multicolumn{2}{|l|}{ Gender } \\
\hline Male & $87(56 \%)$ \\
\hline Female & $68(44 \%)$ \\
\hline \multicolumn{2}{|l|}{ Ethnicity } \\
\hline Caucasian & $130(84 \%)$ \\
\hline Mixed & $14(9 \%)$ \\
\hline African-American & $8(5 \%)$ \\
\hline Hispanic & $3(2 \%)$ \\
\hline \multicolumn{2}{|l|}{ Patient location } \\
\hline Outpatient & $133(86 \%)$ \\
\hline Inpatient & $22(14 \%)$ \\
\hline \multicolumn{2}{|l|}{ Insurance (133 cases) } \\
\hline Private & $90(68 \%)$ \\
\hline Medicaid & $43(32 \%)$ \\
\hline \multicolumn{2}{|l|}{ Dysmorphism (154 cases) } \\
\hline Yes & $73(47 \%)$ \\
\hline Mild & $17(11 \%)$ \\
\hline No & $64(42 \%)$ \\
\hline \multicolumn{2}{|l|}{ OFC } \\
\hline Normal & $93(61 \%)$ \\
\hline$<-1.88$ SD & $42(28 \%)$ \\
\hline$>+1.88 \mathrm{SD}$ & $17(11 \%)$ \\
\hline \multicolumn{2}{|l|}{ Height } \\
\hline Normal & $99(64 \%)$ \\
\hline$<5$ th percentile & $50(32 \%)$ \\
\hline$>95$ th percentile & $6(4 \%)$ \\
\hline \multicolumn{2}{|l|}{ Weight } \\
\hline Normal & $106(68 \%)$ \\
\hline$<5$ th percentile & $36(23 \%)$ \\
\hline$>95$ th percentile & $13(8 \%)$ \\
\hline Consanguinity & $6(3.9 \%)$ \\
\hline Average age at ES (range) & 6 years ( 3 days to 33 years) \\
\hline Average turnaround time in months (range) & $4.7(1.3-7.9)$ \\
\hline
\end{tabular}


were either paid for by philanthropic support or absorbed by the hospital that sent the testing. Out-of-pocket costs to families with private insurance and for whom ES was sent as outpatients were available for 82 cases (Figure 1a). Fifty-four of these cases had an out-of-pocket cost of $\$ 0$, and the average cost was $\$ 386.31$; the maximum cost was $\$ 4,012$.

The average age at which symptoms in patients began was 11 months, with a median of 7 weeks, ranging from birth to
22 years. Of note, 63 patients (41\%) had onset of symptoms at birth. Patients were first seen by a medical geneticist at an average age of 3 years, with a median of 14 months and a range from birth to 31 years old.

The primary indications for ES, the most commonly affected organ systems, and the most common neurodevelopmental findings are presented in Figure 1 $\mathbf{b}-\mathbf{d}$, respectively. The average number of organ systems affected in our cohort was 2.6

a
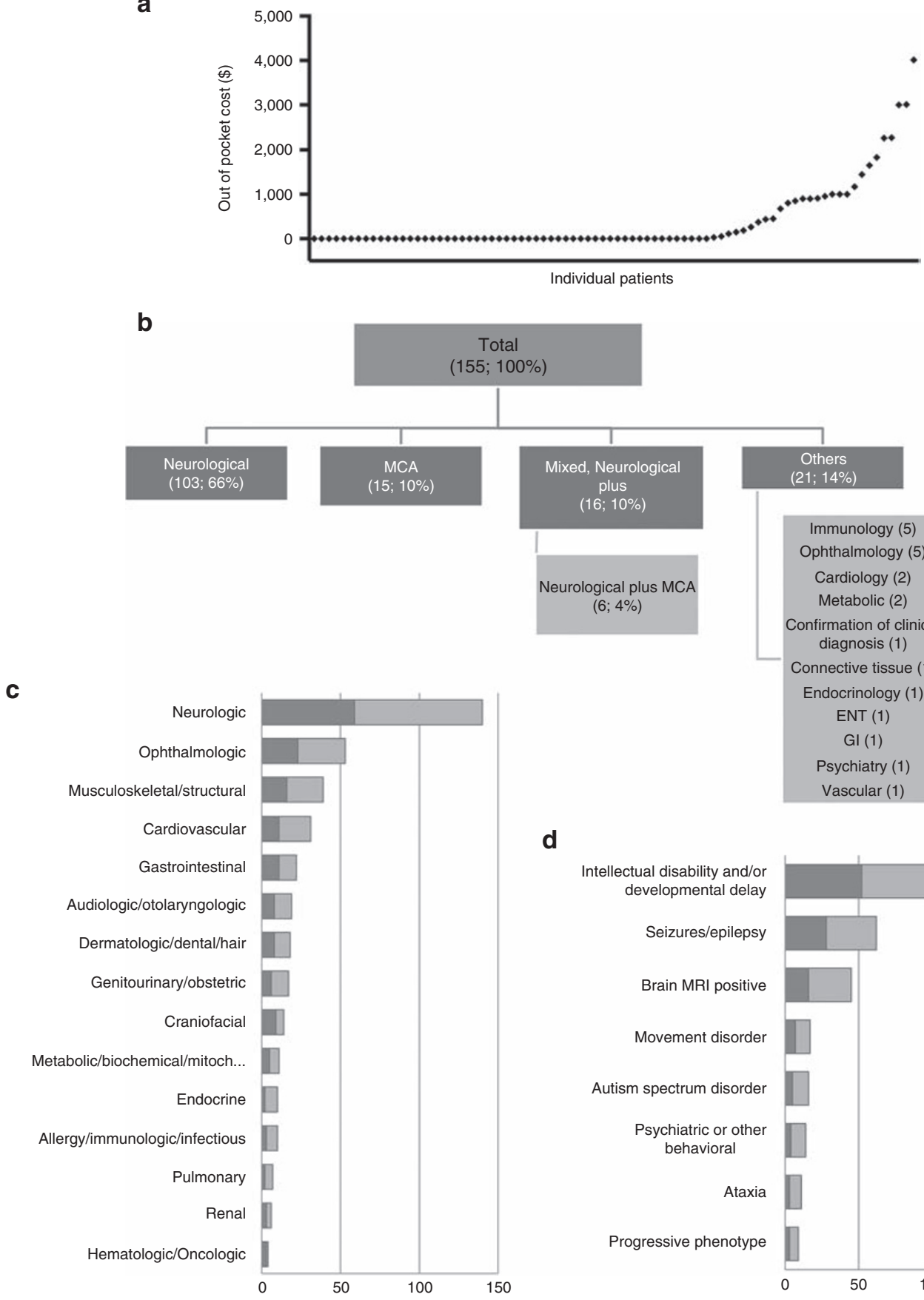
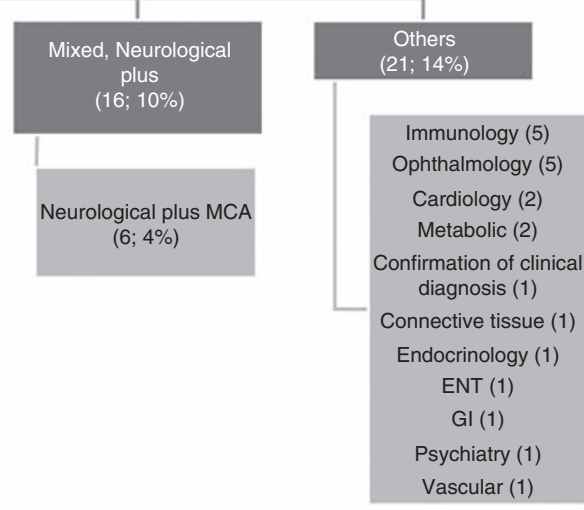

d

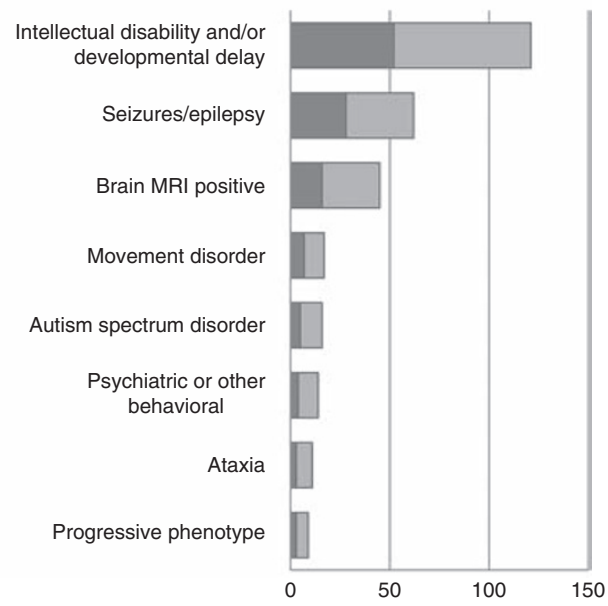

Figure 1 Cost and phenotypic characterization of the cohort. (a) Scatter plot of the out-of-pocket cost in ascending order. (b) Each case was assigned a phenotype-based, single, primary indication for performing ES. The number and percentage of cases are shown in parentheses. MCA, multiple congenital anomalies. (c) Each phenotypic feature of the probands was assigned to an organ system, and the total count of cases is displayed. (d) The frequency and distribution of the neurodevelopmental phenotypes in the cohort. The darker portion of the bar in $\mathbf{c}$ and $\mathbf{d}$ indicates the proportion of cases with a definitive diagnosis. 
(median, 2; range, 1 to 7 out of 15 possible organ systems). The average number of services (other than genetics) involved in the care of the patients in our cohort was 3.3 (median, 3; range, 0 to 10 out of 19 possible services).

\section{Variant classification and interpretation}

The diagnostic laboratory reported 237 genetic variants, with an average of 1.5 variants reported per patient and a range from 0 to 6 . The distribution of genetic variants based on variantlevel assertion was as follows: 79 pathogenic, 37 likely pathogenic, 107 VUS, and 14 incidental findings (Supplementary Figure S2 online, Supplementary Tables S1 and S2 online) that were classified by the laboratory as known pathogenic (12) or expected pathogenic (2). Among the 155 cases, 56 cases (36\%) had a definitive diagnosis based on case-level assertion by the laboratory, 60 cases were reported as possible, 10 cases were reported as candidate, and 29 cases were reported as negative (Figure 2a, Supplementary Figure S1 online, Supplementary Tables S3 online). Due to the presence of autosomal recessive (AR) conditions and blended phenotypes among the 56 definitive cases, the number of variants was 71. Definitive diagnoses in four genes were identified in more than one unrelated case: ARID1B (2), GABRB2 (3), NGLY1 (2), and PTPN11 (2). Eleven cases had mitochondrial genome sequencing completed as part of the ES order, but none of these yielded abnormal results. Misattribution or nonpaternity was found in two families as a result of ES testing.

Based on the assessment of the ordering medical geneticist, the final diagnosis was changed for 21 subjects (14\%) (Figure 2b, Supplementary Figures S1 and S2 online, Supplementary Tables S1, S2, and S3 online; Table 2; Supplementary Table S7 online). The diagnosis for 16 subjects was promoted such that the clinical geneticist determined that the variant was more definitively related to the phenotype; for 5 subjects, it was

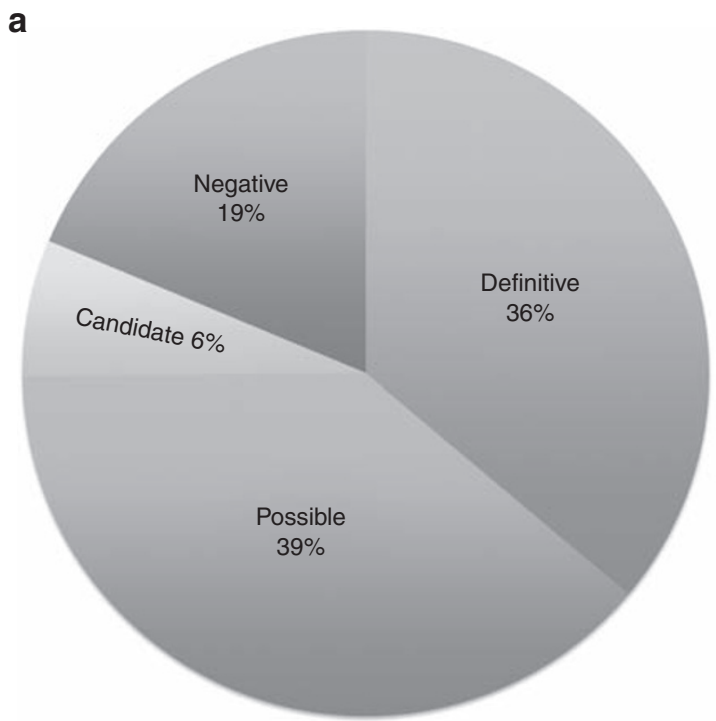

demoted. Consequently, there was a net gain of 11 additional definitive diagnoses, for a total of 67 cases (43\%) definitively diagnosed (Supplementary Table S7 online). There were multiple reasons for changing the case-level classification (Table 2). First, the clinical geneticist has direct and detailed knowledge of the patient's phenotype and the opportunity to order followup studies including biochemical and radiological studies, segregation analysis of relatives, and/or single-gene resequencing or deletion/duplication studies to search for a mutation in the second allele. Furthermore, there were variants in candidate genes that were promoted because of subsequent publication of new syndromes, either in other similarly affected patients or by the contribution of these patients to syndrome discovery themselves. ${ }^{11-16}$ Thirty-two (48\%) of the 67 definitive cases had mutations in genes described in 2011 or later. This includes seven (10\%) described as new genetic syndromes ${ }^{12-16}$ (WES038, WES052, WES057, WES062, WES079, WES105, WES121), three of which are in the process of being published. Five cases (7.5\%) had definitive variants in two genes resulting in "blended phenotypes" (WES028 (ref. ${ }^{17}$ ), WES030, WES060, WES070, WES128). Reanalysis of the exome data was performed for 14 cases by the molecular laboratory, usually 12 to 18 months after the initial report was generated. In seven cases, the reanalysis resulted in no change; in four cases, it resulted in a new definitive diagnosis (WES013, WES019, WES039, WES131 (ref. ${ }^{18}$ )) due to subsequently published new syndromes or functional analysis of variants. In one case, a previously reported variant was demoted (WES002). The remaining two cases (WES099, WES112) involved efforts by the laboratory to identify candidate disease genes for which there have not yet been human phenotypes associated.

We then assessed the relationship between the diagnostic yield, as determined by the medical geneticist, and various demographic and phenotypic characteristics (Supplementary

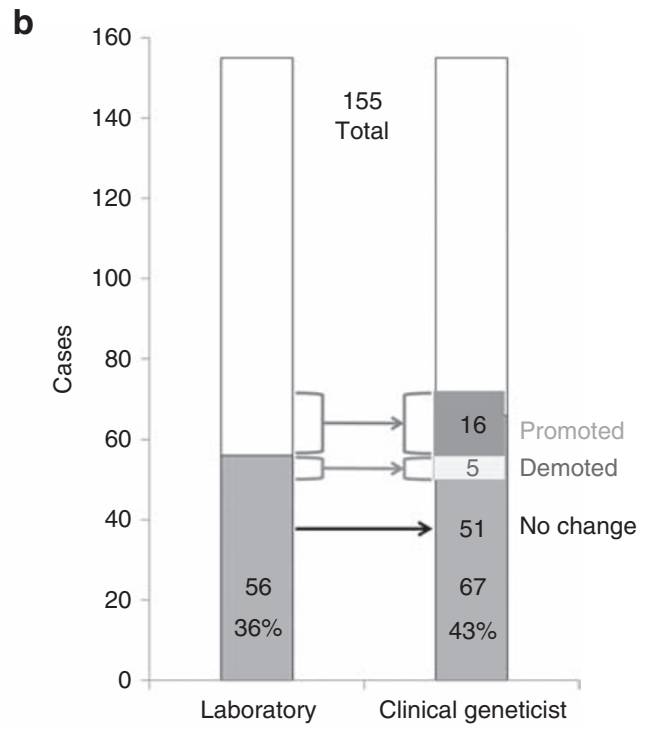

Figure 2 Characterization of case-level and clinical-level assertions. (a) The relative percentages of each case-level classification as reported by the testing laboratory. (b) The diagnostic rates according to case-level and clinical-level assertions are shown as the proportion of cases in gray. The change in classification of cases is indicated, with 16 cases promoted and 5 demoted. 


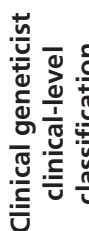

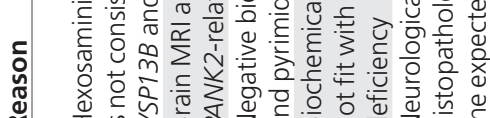

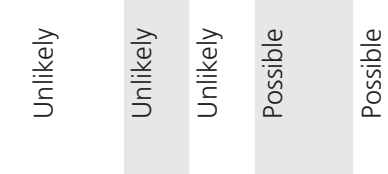

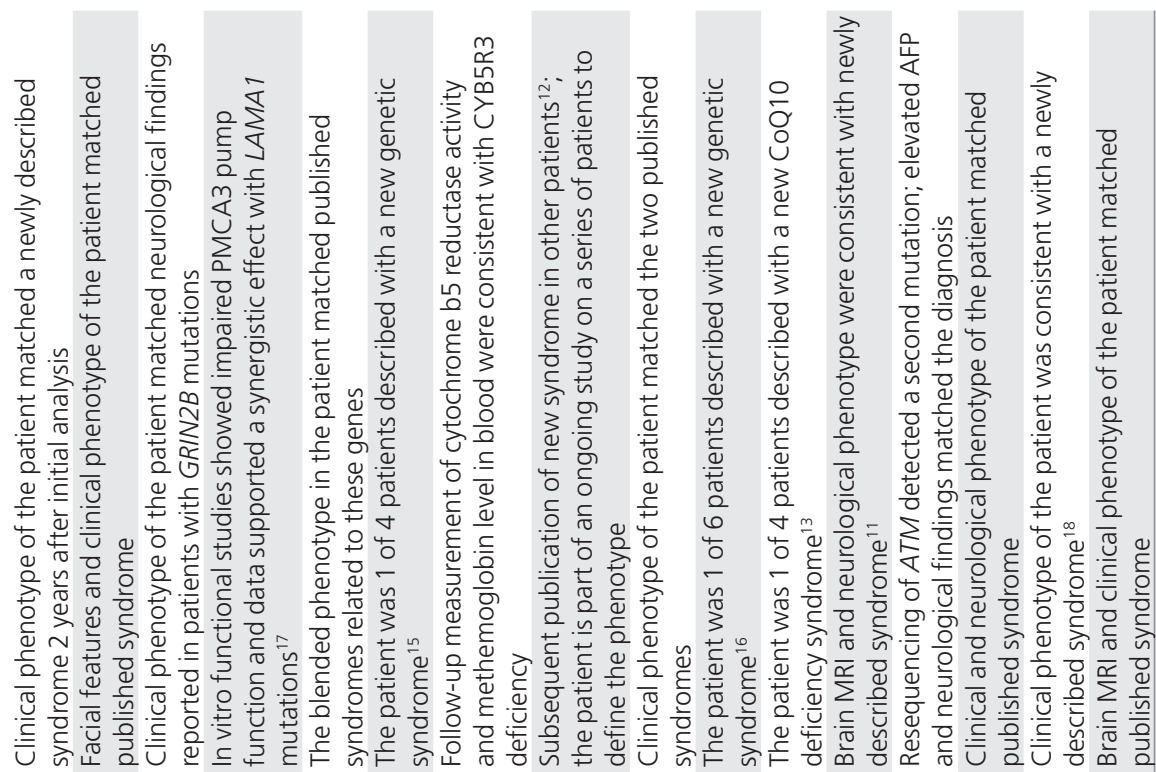

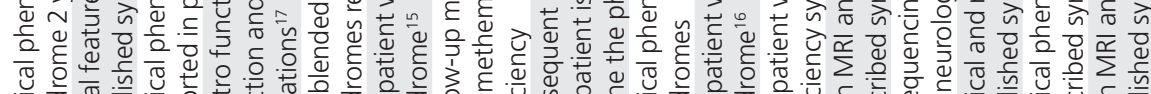

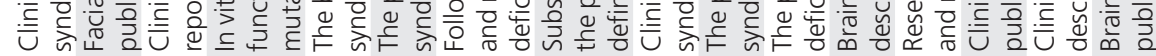

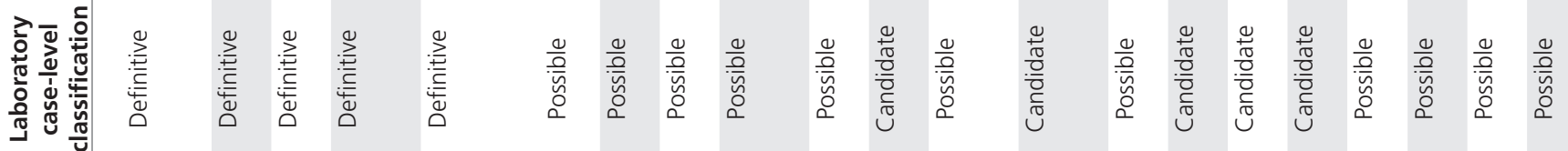

ำ 竞

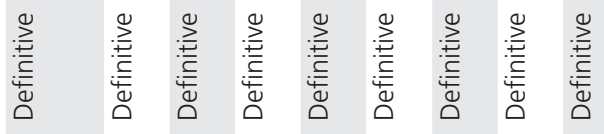
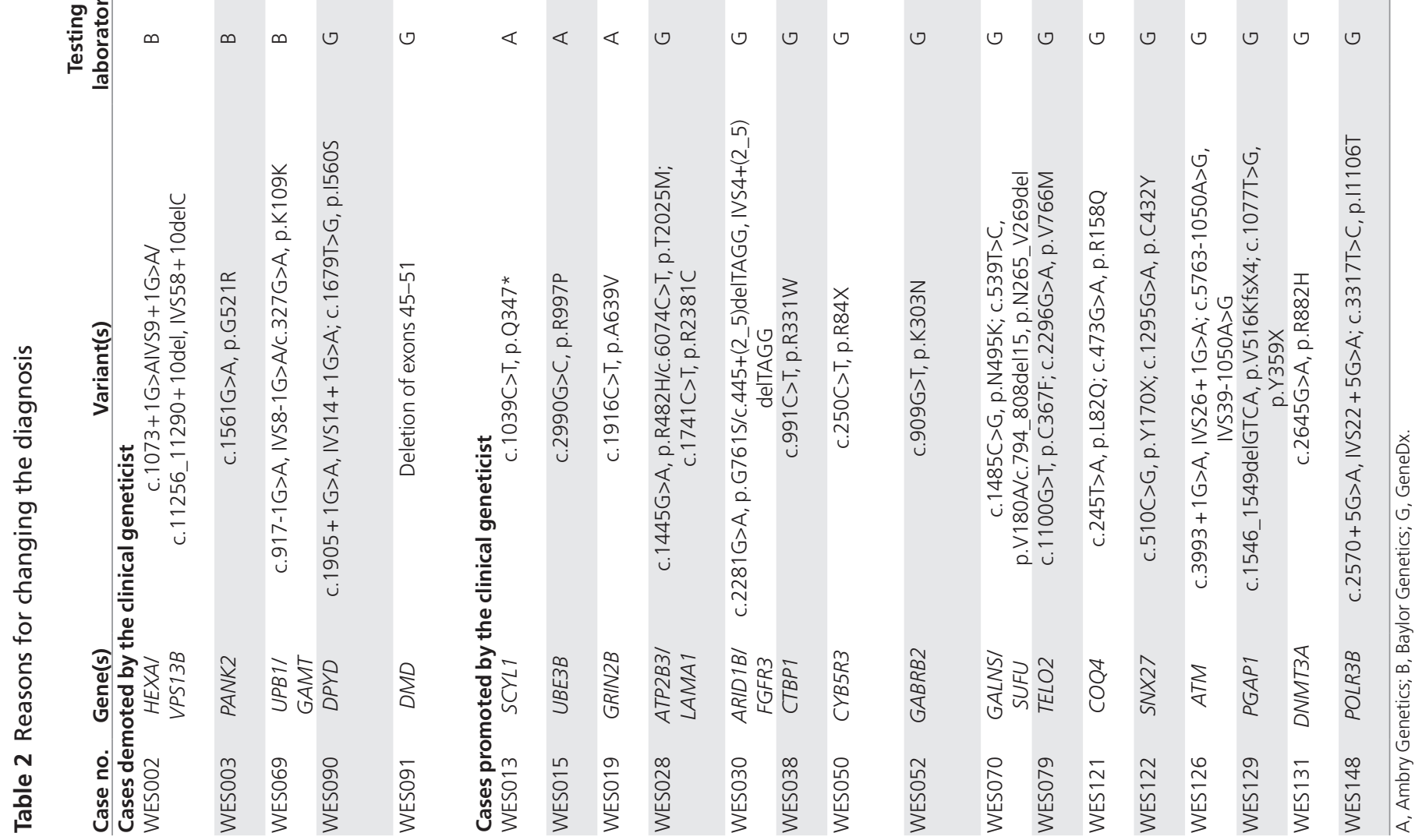
Table S4 online). Our results indicated a higher diagnostic yield for females (47\%), patients with a craniofacial anomaly (64\%), and patients with an abnormal head circumference, specifically microcephaly (50\%), but none of these effects was statistically significant. Caucasians had a statistically significant higher rate of diagnosis compared to all other racial groups (46 vs. $24 \%, P$ $=0.04)$ that persisted after adjusting for craniofacial anomaly in the multivariable logistic regression model, demonstrating the disproportionately low diagnosis rate for non-Caucasians. The following additional categories were tested for effects on diagnostic rates and were found to be not significant: inpatient versus outpatient status, all other phenotypic categories, death, abnormal height or weight, dysmorphism, and positive family history.

The inheritance patterns in the 72 conditions ( 67 subjects; 5 with 2 conditions caused by variants in different genes) that were determined to be definitive were as follows: 42 (58\%) autosomal dominant (AD), 24 (33\%) AR, and 6 (8\%) X-linked. Of the 89 variants that are associated with these 72 conditions, 34 (38\%) were de novo, including one variant in each of two cases with AR conditions (Supplementary Table S7 online). The average paternal age at delivery of the 42 patients with de novo mutations was 32 years, with a median age of 32 years and a range of 22 to 49 years. For the inherited variants, 25 were passed from the mother, 18 from the father, 4 from both (homozygous for recessive condition), and 8 had unknown inheritance due to at least 1 parent not being sequenced. We observed reduced penetrance of five variants that were associated with $\mathrm{AD}$ conditions and inherited from seemingly unaffected parents, although parental cardiac evaluations are pending in two of these cases.

In nine cases, ES was sent prior to the implementation of the 2013 American College of Medical Genetics and Genomics (ACMG) guidelines for reporting incidental findings. ${ }^{19}$ Of the remaining 146 cases, 5 (3\%) families opted out and 141 (97\%) families elected to receive the findings. Fourteen patients (10\%) had one incidental finding each. Incidental findings were found in the following genes from the ACMG-recommended list of 56 genes: BRCA2 (2), FBN1, LDLR (2), MYBPC3 (4), MYH7, RET, SCN5A, and TTN (2) (Supplementary Table S5 online). Although the laboratories' reports indicate that these incidental variants are known pathogenic in 12 cases, only 5 of these 12 are uniformly classified as pathogenic in ClinVar (http://www.ncbi. nlm.nih.gov/clinvar/) and the remainder have conflicting interpretations of pathogenicity, with some submitters even identifying 2 of these variants as likely benign (Supplementary Table S5 online). Follow-up assessment or evaluation was performed based on established guidelines and protocols for these cases and their carrier relatives (Supplementary Table S5 online).

\section{The effect of exome results on auxiliary tests, management, and research studies}

We investigated whether the exome results affected subsequent diagnostic work-up or changed patient management. Additional diagnostic studies were performed for 84 subjects (54\%), including molecular studies (proband or family members) for 37 (24\%), imaging studies for 29 (19\%), and biochemical and/or chemistry tests for 22 (14\%). The distribution of the 84 cases based on clinical-level assertion was as followings: 48 were definitive, 4 were likely, 8 were possible, 20 were unlikely, 1 was incidental only, and 3 had completely negative results but had follow-up genetic testing performed due to concerns regarding poor coverage of the exome data at particular genes of interest (Supplementary Material 1 online, Supplementary Table S6 online). In 12 of the 84 cases, these follow-up studies were due to the discovery of an ACMG-designated incidental finding. An echocardiogram was performed for 19 (12\%) probands or family members, 7 of which were due to incidental findings. In addition, cancer surveillance protocols were initiated in 7 probands or related family members due to variants found by ES, 2 of which were incidental. Three families used the ES information for prenatal or preimplantation genetic diagnosis.

In 8 of the 67 definitive cases (12\%), clinical care was directly altered due to primary ES findings as follows: (i) discontinuation of levothyroxine (WES113, SLC16A2); (ii) cardiac ablation in an asymptomatic patient (WES118, TBX3) found to have Wolff-Parkinson-White syndrome on the EKG that was ordered based on ES results; (iii) prophylactic thyroidectomy and Hirschprung's diagnosis (WES018, RET); (iv) neuropsychology evaluation because of known deficits associated with this condition, although not obviously present in this case that showed ADHD and anxiety disorder and resulted in an atomoxetine prescription (WES057, WAC); (v) orthopedics referral of a patient (WES025, PHF6) with a condition known to cause musculoskeletal phenotypes that led to diagnosis and surgical repair of her scoliosis; (vi) amantadine trial initiated for ataxia telangiectasia (WES126, ATM); (vii) a trial of methylene blue and vitamin $\mathrm{C}$ in a patient (WES050, CYB5R3) with methemoglobinemia; and, finally, (viii) serine prescription for serineresponsive seizures (WES059, PHGDH). Thirty-six patients were enrolled in research studies related to their ES results. These involved efforts to characterize the potential functional effect of a particular variant and reanalysis of otherwise negative clinical exome data for research purposes.

\section{DISCUSSION}

Although several studies have reported clinical ES results, most of these reports have come from diagnostic laboratories and do not focus on the medical geneticists' interpretations of the findings. The main purpose of the present study was to evaluate the medical geneticist's role in the optimal interpretation of the exome results and how this might alter the final diagnostic yield. The overall definitive diagnosis rate of clinical ES in our cohort was $36 \%$ based on laboratory sequencing data, but this increased to $43 \%$ after the integration of the molecular and phenotypic data by the medical geneticist and the incorporation of additional diagnostic modalities. Fifty-four percent of patients in our cohort underwent "postanalytical" auxiliary diagnostic studies, including biochemical analyses, imaging studies, complementary molecular tests (e.g., deletion and duplication analysis of a specific gene or Sanger sequencing of a gene with low 
exome coverage), and/or genotyping affected and unaffected family members for segregation analysis.

Furthermore, each genetic variant was evaluated by a thorough literature review and searching databases such as ExAC (Exome Aggregation Consortium) and ClinVar. This extensive postexome assessment by the clinician is time-consuming and illustrates that ES results as reported by the molecular laboratory require clinical context. The laboratory identifies sequence changes and provides information about suspected pathogenicity, but the medical geneticist must compare the expected phenotype associated with the molecular finding to the patient's phenotype to determine if they align and whether the molecular finding may account for the patient's clinical presentation. In five cases, we determined that the molecular finding was not consistent with the patient's phenotype and that the genetic variant was considered to be either benign or not completely explanatory. In 16 other cases, the classification was promoted to a more definitive category and, ultimately, the final diagnosis was modified (Table 2). However, in other patients the final diagnosis is still uncertain and pathogenicity of the variants is difficult to establish due to lack of functional data, inability to perform segregation analysis, incomplete explanation of the phenotype by the variant, or candidate gene status. These limitations pose challenges to the clinician and demonstrate that receiving the exome results can be the beginning of a continuing exploration process rather than the end of the "diagnostic odyssey."

As evidenced by large-scale research studies that use ES as a tool for discovery such as the Deciphering Developmental Disorders study, ${ }^{20}$ the rate of discovery of new genetic syndromes is rapidly increasing. Therefore, reanalysis of previously reported clinical ES data has the potential to increase the sensitivity of the test. In fact, $48 \%$ of definitive cases in our study had mutations in genes with associated syndromes described in 2011 or later. Subsequent reanalysis of the exome data, either at the request of the medical geneticist or at the prompting of internal reanalysis by the diagnostic laboratory, directly resulted in seven additional definitive diagnoses than would have otherwise been obtained, illustrating the need to perform ongoing data mining for previously submitted cases with negative exome results.

The increased diagnostic yield in our cohort relative to previously reported clinical series ${ }^{3-6,8-10}$ can be partly attributed to the selection process we apply for subspecialty referrals for the Exome Clinic, including an ES-specific referral form (Supplementary Material 2 online) and review of the suitability of the case by a medical geneticist. It is also possible that there was a selection bias toward the most severely affected patients referred to a tertiary medical center, reflected by a relatively high number of organ systems, services involved, highly skewed growth parameters, and high rate of dysmorphism in the probands when the test was initially implemented in our institution. We cannot exclude the contribution of other factors such as a high trio rate (83\%), different categories of indications, or differences in sample size.
This study has a number of important limitations. For example, ES was ordered through three laboratories, each of which used a different terminology to classify the variants in relation to patient's phenotype, which limits cross-case comparison. In addition, the laboratories' data analysis processes changed over time as algorithms have improved and ACMG guidelines have been implemented. However, there were no statistically significant differences among the three different diagnostic laboratories regarding the number of cases with incidental findings, the proportion of cases with a definitive diagnosis at the case-level, and whether the case-level classification was revised by the clinician (Supplementary Material 1 online). Another factor limiting the generalizability of our findings is that these patients were all part of a highly selected population that was evaluated at a tertiary medical center.

Our study shows that clinical ES is a powerful diagnostic tool, especially for atypical and mild presentations of well-established genetic syndromes. For example, none of the patients who received diagnoses of CHARGE, Noonan, ataxia telangiectasia, and LADD syndromes met clinical diagnostic criteria; instead, they exhibited partial phenotypes. Furthermore, the discovery of five patients in our cohort with "blended phenotypes," as similarly described in other cohorts, ${ }^{3,21,22}$ should change our traditional diagnostic approach. ES is a valuable gene discovery tool, as illustrated in seven patients who were included in initial case series that described novel genetic syndromes. Other unexpected exome results were related to potential germline mosaicism in one case (WES057) and uniparental disomy in another case (WES050). This information about non-Mendelian modes of inheritance was very important for providing accurate recurrence risks for future pregnancies. ES also uncovered nonpaternity in two cases, which required a consultation with our institutional ethics committee and ultimately led to altered strategies for pretest counseling regarding this complicated issue.

Incidental findings present in $9 \%$ of our cohort patients often resulted in additional interventions in both the probands and their carrier relatives. This number is higher than we would have expected by comparison to previous cohorts. ${ }^{3-5,23}$ However, based on the conflicting assertions in ClinVar (Supplementary Table S5 online), it is clear that the performing laboratories over-called incidental findings and that the actual rate is 3.8\% $(6 / 14)$. These data illustrate the challenges in variant classification and the need for simple and consistent criteria for classification based on variant-specific databases and knowledge bases. ${ }^{23}$ We speculate that this lack of uniformity may be due to changes in how variants are classified over time, especially after the release of the 2015 ACMG guidelines. ${ }^{24}$ The role of the medical geneticist in following-up these incidental results is as important as it is for following-up primary results because subsequent monitoring, such as cancer screening and cardiac monitoring, can have lifesaving consequences for the patients and their relatives. However, the conflicting interpretations of the data as presented here and the workup performed for patients with uncertain incidental findings (Supplementary Table S5 


\section{ORIGINAL RESEARCH ARTICLE}

online) illustrate the challenges that medical geneticists face and reveal one of the significant drawbacks of ES related to false-positive incidental findings, which could lead to substantial harmful consequences, including performing unnecessary and potentially harmful tests and procedures, increased healthcare costs due to performing unnecessary follow-up evaluations, and causing anxiety among a percentage of patients undergoing ES. ${ }^{23,25,26}$ These are important points that should be carefully considered prior to ordering ES and during pretesting counseling.

For many patients, ending a diagnostic odyssey limits additional expensive, time-consuming, and potentially invasive diagnostic procedures. It also allows precise determination of recurrence risk and prognosis. ES results were used by three families from our cohort for prenatal diagnosis testing. Although the discovery of a treatable condition can dramatically change the clinical outcome, the exome resulted in specific treatments in only a limited number of our patients. Nevertheless, clinical management was directly altered due to primary ES findings in eight patients, comprising $5.2 \%$ of all patients who underwent ES. It is also possible that careful clinical assessment for part of these cases would detect clinical findings that might ultimately change the management even without the molecular data.

The correlation of diagnostic yield in our cohort with various demographic and phenotypic characteristics showed a higher yield for Caucasians, females, patients with craniofacial anomalies, and patients with abnormal head circumference, but none of these reached statistical significance except for ethnic background (Supplementary Material 1 online, Supplementary Table S4 online). It is important to note that patients from minority populations are under-represented in our cohort, suggesting a need for increased access to ES for individuals from these backgrounds. Although the average out-of-pocket cost for ES was $\$ 386$ per family, and although we do not have detailed socioeconomic data for our cohort, we speculate that economic factors may play a role in this discrepancy. Publicly funded insurance plans do not routinely provide coverage for ES, and families with high out-of-pocket costs sometimes selfselected not to pursue this testing. Compounding this situation, non-Caucasians achieved a significantly lower diagnostic rate of only $24 \%$. This finding may be due, in part, to an underrepresentation of minority populations in variant databases, causing challenges in interpreting the clinical significance of variants found in these populations.

Taking into account the work involved in interpreting and following-up both primary and incidental exome findings, the complex phenotype of patients referred for ES, as well as the constantly evolving nature of these results due to reanalysis and publication of new genetic syndromes, medical geneticists serve an essential role in this complex diagnostic process. This study shows that the partnership of the clinician with the molecular laboratory can increase the diagnostic yield by $7 \%$. An accurate molecular diagnosis ends a diagnostic odyssey, allows for precise genetic counseling, and has the potential to change clinical management. It is also the launching point for the development of targeted pharmacologic therapies, which can hopefully translate these discoveries into efficacious novel treatments to achieve the promise of personalized genomic medicine.

\section{SUPPLEMENTARY MATERIAL}

Supplementary material is linked to the online version of the paper at http://www.nature.com/gim

\section{ACKNOWLEDGMENTS}

D.B. is supported by the National Institute of Mental Health of the National Institutes of Health (NIH) under award T32MH014677 and the National Heart, Lung, and Blood Institute of the $\mathrm{NIH}$ under award K12HL120002. M.V. is partially supported by the Eunice Kennedy Shriver National Institute of Child Health \& Human Development of the NIH under award U54HD087011 and the Intellectual and Developmental Disabilities Research Center at Washington University.

\section{DISCLOSURE}

D.B.'s and M.V.'s work haves been funded by the NIH. The other authors declare no conflict of interest.

\section{REFERENCES}

1. Biesecker LG, Green RC. Diagnostic clinical genome and exome sequencing. $N$ Engl J Med 2014;370:2418-2425.

2. Bamshad MJ, Ng SB, Bigham AW, et al. Exome sequencing as a tool for Mendelian disease gene discovery. Nat Rev Genet 2011;12:745-755.

3. Yang Y, Muzny DM, Xia F, et al. Molecular findings among patients referred for clinical whole-exome sequencing. JAMA 2014;312:1870-1879.

4. Lee $H$, Deignan JL, Dorrani $N$, et al. Clinical exome sequencing for genetic identification of rare Mendelian disorders. JAMA 2014;312:1880-1887.

5. Farwell KD, Shahmirzadi L, El-Khechen D, et al. Enhanced utility of familycentered diagnostic exome sequencing with inheritance model-based analysis: results from 500 unselected families with undiagnosed genetic conditions. Genet Med 2015;17:578-586.

6. Retterer K, Juusola J, Cho MT, et al. Clinical application of whole-exome sequencing across clinical indications. Genet Med 2016;18:696-704.

7. Hennekam RC, Biesecker LG. Next-generation sequencing demands nextgeneration phenotyping. Hum Mutat 2012;33:884-886.

8. Shashi V, McConkie-Rosell A, Schoch K, et al. Practical considerations in the clinical application of whole-exome sequencing. Clin Genet 2016;89:173-181.

9. Iglesias A, Anyane-Yeboa K, Wynn J, et al. The usefulness of whole-exome sequencing in routine clinical practice. Genet Med 2014;16:922-931.

10. Valencia CA, Husami A, Holle J, et al. Clinical impact and cost-effectiveness of whole exome sequencing as a diagnostic tool: a pediatric center's experience. Front Pediatr 2015;3:67.

11. Damseh N, Danson CM, Al-Ashhab M, et al. A defect in the retromer accessory protein, SNX27, manifests by infantile myoclonic epilepsy and neurodegeneration. Neurogenetics 2015;16:215-221.

12. Srivastava $S$, Cohen J, Pevsner J, et al. A novel variant in GABRB2 associated with intellectual disability and epilepsy. Am J Med Genet A 2014;164A:2914-2921.

13. Chung WK, Martin K, Jalas C, et al. Mutations in COQ4, an essential component of coenzyme $Q$ biosynthesis, cause lethal neonatal mitochondrial encephalomyopathy. J Med Genet 2015;52:627-635.

14. DeSanto C, D'Aco K, Araujo GC, et al. WAC loss-of-function mutations cause a recognisable syndrome characterised by dysmorphic features, developmental delay and hypotonia and recapitulate 10p11.23 microdeletion syndrome. J Med Genet 2015;52:754-761.

15. Beck DB, Cho MT, Millan F, et al. A recurrent de novo CTBP1 mutation is associated with developmental delay, hypotonia, ataxia, and tooth enamel defects. Neurogenetics 2016;17:173-178.

16. You J, Sobreira NL, Gable DL, et al. A syndromic intellectual disability disorder caused by variants in TELO2, a gene encoding a component of the TTT complex. Am J Hum Genet 2016;98:909-918. 
17. Calì T, Lopreiato R, Shimony J, et al. A novel mutation in isoform 3 of the plasma membrane Ca2+ pump impairs cellular Ca2+ homeostasis in a patient with cerebellar ataxia and laminin subunit $1 \alpha$ mutations. J Biol Chem 2015;290:16132-16141.

18. Tatton-Brown K, Seal S, Ruark E, et al.; Childhood Overgrowth Consortium. Mutations in the DNA methyltransferase gene DNMT3A cause an overgrowth syndrome with intellectual disability. Nat Genet 2014;46:385-388.

19. Green RC, Berg JS, Grody WW, et al.; American College of Medical Genetics and Genomics. ACMG recommendations for reporting of incidental findings in clinical exome and genome sequencing. Genet Med 2013;15:565-574.

20. Akawi N, McRae J, Ansari M, et al.; DDD study. Discovery of four recessive developmental disorders using probabilistic genotype and phenotype matching among 4,125 families. Nat Genet 2015;47:1363-1369.

21. Posey JE, Rosenfeld JA, James RA, et al. Molecular diagnostic experience of whole-exome sequencing in adult patients. Genet Med 2016;18:678-685.
22. Li Y, Salfelder A, Schwab KO, et al. Against all odds: blended phenotypes of three single-gene defects. Eur J Hum Genet 2016;24: 1274-1279.

23. Amendola LM, Dorschner MO, Robertson PD, et al. Actionable exomic incidental findings in 6503 participants: challenges of variant classification. Genome Res 2015;25:305-315.

24. Richards S, Aziz N, Bale S, et al.; ACMG Laboratory Quality Assurance Committee. Standards and guidelines for the interpretation of sequence variants: a joint consensus recommendation of the American College of Medical Genetics and Genomics and the Association for Molecular Pathology. Genet Med 2015;17:405-424.

25. Biesecker LG. Overcalling secondary findings. Genet Med 2016;18:416.

26. Manrai AK, Funke BH, Rehm HL, et al. Genetic misdiagnoses and the potential for health disparities. N Engl J Med 2016;375:655-665. 